\title{
Eclipse Prediction and the Length of the Saros in Babylonian Astronomy
}

\author{
LIS BRACK-BERNSEN*AND JOHN M. STEELE ${ }^{\dagger}$
}

\begin{abstract}
The Saros cycle of 223 synodic months played an important role in Late Babylonian astronomy. It was used to predict the dates of future eclipse possibilities together with the times of those eclipses and underpinned the development of mathematical lunar theories. The excess length of the Saros over a whole number of days varies due to solar and lunar anomaly between about 6 and $9 \mathrm{~h}$. We here investigate two functions which model the length of the Saros found in Babylonian sources: a simple zigzag function with an 18-year period presented on the tablet BM 45861 and a function which varies with the month of the year constructed from rules found on the important procedure text TU 11. These functions are shown to model nature very well and to be closely related. We further conclude that these functions are the likely source of the Saros lengths used to calculate the times of predicted eclipses and were probably known by at latest the mid-sixth-century BC.
\end{abstract}

\section{Eclipse Prediction and the Saros}

Throughout most, perhaps all, of the Late Babylonian period, the basic method of predicting eclipses of the sun and moon in Babylonia was based upon the so-called Saros cycle (SC) of 223 synodic months. This method was not restricted to simply identifying dates 1 or more Saroi after a visible eclipse but could be used to identify the dates of all eclipse possibilities (EPs). It almost certainly originated with lunar eclipses and was then applied to solar eclipses. Indeed, it is most likely that all Babylonian eclipse theories-for want of a better term - were developed from lunar eclipse observations and used for predicting lunar eclipses and then applied by analogy to solar eclipses. The method for predicting lunar eclipses itself arises by combining the Saros with the realization that EPs generally occur every 6 months but occasionally after only 5 months. Therefore, if within a Saros of 223 months, there are $a$ eclipses at a 6-month interval and $b$ eclipses at a 5-month interval, simple mathematics shows from $6 a+5 b=223$ that $a=33$ and $b=5$. Within a Saros of 223 months, there are therefore 38 EPs, of which 33 are at a 6-month interval and the remaining 5 are at a 5-month interval. Distributing the 5-month intervals as evenly as possible, we obtain five groups of eclipses in which the first eclipse of each group

\footnotetext{
*Wissenschaftsgeschichte, Institut für Philosophie, Universität Regensburg, D-93040 Regensburg, Germany.

${ }^{\dagger}$ Department of Physics, University of Durham, Durham, United Kingdom.
} 
follows a 5-month interval and the remainder are at 6-month intervals. The groups contain, respectively, eight, seven, eight, seven, and eight eclipses. Since EPs repeat after 1 Saros, this simple distribution results in a matrix-like scheme by which all EPs can be predicted. All that needs to be done is to decide which EP corresponds to the first eclipse in the first column of the matrix. ${ }^{1}$

It is not known exactly when this scheme for predicting eclipses was developed, but it was almost certainly before about 575 BC. The tablet LBAT * 1420, published as Text No. 6 in Hunger (2001), contains a collection of eclipse observations for the period from at least $604 \mathrm{BC}$ to $576 \mathrm{BC}$, and there is nothing to suggest that the text was compiled significantly after the date of its contents. Interspersed between the observed eclipses, the months of EPs are also noted. The distribution of EPs is exactly what we would expect from the Saros scheme described above and thus implies that scheme was in use by at least $575 \mathrm{BC}$. It may well have been used earlier. The earliest eclipse records we possess apparently begin in 747 $\mathrm{BC},{ }^{2}$ and already they include predicted eclipses. Furthermore, we have three tablets that originally formed part of a large compilation of lunar eclipse observations and predictions covering 24 Saroi. $^{3}$ The earliest preserved eclipse on these tablets was in $731 \mathrm{BC}$, but it is very probable from the structure of the compilation that when complete, it covered the period from $747 \mathrm{BC}$ to $315 \mathrm{BC}$. The compilation is set out in exactly the form of the Saros scheme matrix described above, and predictions are given of the times of all EPs that were not observed. The structure of the large compilation as reconstructed by Steele (2000a) from other extant eclipse records is shown in the large scheme below (Table 1).

Of course, this compilation does not tell us anything about when the Saros scheme was brought into use, since it is quite possible that some of the 'predicted' eclipses were calculated backwards when the text was compiled to fill it out. Nevertheless, we have the evidence from LBAT $^{*} 1420$ that the Saros scheme was being used by at least ca 575 BC.

Determining which months are EPs is not the end of the matter, however. Many of the extant records of eclipse predictions also include the time of day or night when the eclipse was expected to occur. As has been shown by Steele and Stephenson (1997), this time relates to the moment when the eclipse was expected to begin. This in itself indicates that the times were probably calculated by means of the Saros. If mathematical astronomy had been used to determine the time of the eclipse, it would naturally result in the time of syzygy. To obtain the moment when the eclipse began, a correction to this time would have to be made based upon the expected duration of the eclipse, but with the possible exception of one column of numbers on a single text (the so-called Text $\mathrm{S}$, see below), ${ }^{4}$ no functions for the duration of an eclipse are known from the mathematical astronomical texts. However, when an eclipse was observed, the time when it began was recorded. Adding on the Saros length to this time, the time of the predicted eclipse would also be for when it was expected to begin. ${ }^{5}$

The length of the Saros depends upon the positions of the sun and moon in their cycles of anomalistic motion and varies between about 1,35 and 2,15 uš in excess of an integer 
Table 1. The reconstructed Saros scheme for lunar eclipses.

\begin{tabular}{|c|c|c|c|c|c|c|}
\hline & 1 & 2 & 3 & 4 & 5 & 6 \\
\hline 1 & -746 Feb 6 & -728 Feb 17 & -710 Feb 27 & -692 Mar 10 & -674 Mar 21 & -656 Mar 31 \\
\hline 2 & -746 Aug 2 & -728 Aug 12 & -710 Aug 23 & -692 Sep 3 & -674 Sep 14 & -656 Sep 24 \\
\hline 3 & -745 Jan 26 & -727 Feb 5 & -709 Feb 16 & -691 Feb 27 & -673 Mar 10 & -655 Mar 20 \\
\hline 4 & -745 Jul 22 & -727 Aug 2 & -709 Aug 13 & -691 Aug 23 & -673 Sep 4 & -655 Sep 14 \\
\hline 5 & -744 Jan 15 & -726 Jan 25 & -708 Feb 6 & -690 Feb 16 & -672 Feb 27 & -654 Mar 10 \\
\hline 6 & -744 Jul 11 & -726 Jul 22 & -708 Aug 1 & -690 Aug 12 & -672 Aug 23 & -654 Sep 3 \\
\hline 7 & -743 Jan 3 & -725 Jan 15 & -707 Jan 25 & -689 Feb 6 & -671 Feb 16 & -653 Feb 27 \\
\hline 8 & -743 Jun 30 & $-725 \mathrm{Jul} 11$ & -707 Jul 21 & -689 Aug 2 & -671 Aug 12 & -653 Aug 23 \\
\hline 9 & -743 Nov 25 & -725 Dec 6 & -707 Dec 16 & -689 Dec 28 & -670 Jan 7 & -652 Jan 19 \\
\hline 10 & -742 May 20 & -724 May 31 & -706 Jun 11 & -688 Jun 21 & -670 Jul 2 & -652 Jul 13 \\
\hline 11 & -742 Nov 14 & -724 Nov 25 & -706 Dec 6 & -688 Dec 16 & -670 Dec 28 & -651 Jan 7 \\
\hline 12 & -741 May 10 & -723 May 20 & -705 Мay 31 & -687 Jun 11 & -669 Jun 22 & -651 Jul 2 \\
\hline 13 & -741 Nov 3 & -723 Nov 14 & -705 Nov 25 & -687 Dec 5 & -669 Dec 17 & -651 Dec 27 \\
\hline 14 & -740 Apr 28 & -722 May 10 & -704 May 20 & -686 May 31 & -668 Jun 11 & -650 Jun 22 \\
\hline 15 & -740 Oct 22 & -722 Nov 3 & -704 Nov 13 & -686 Nov 24 & -668 Dec 5 & -650 Dec 16 \\
\hline 16 & -739 Mar 20 & -721 Mar 31 & -703 Apr 10 & -685 Apr 22 & -667 May 2 & -649 May 13 \\
\hline 17 & -739 Sep 12 & -721 Sep 23 & -703 Oct 3 & -685 Oct 15 & -667 Oct 25 & -649 Nov 6 \\
\hline 18 & -738 Mar 9 & -720 Mar 19 & -702 Mar 31 & -684 Apr 10 & -666 Apr 21 & -648 May 2 \\
\hline 19 & -738 Sep 1 & -720 Sep 12 & -702 Sep 23 & -684 Oct 3 & -666 Oct 15 & -648 Oct 25 \\
\hline 20 & $-737 \mathrm{Feb} 26$ & -719 Mar 9 & -701 Mar 20 & -683 Mar 30 & -665 Apr 10 & -647 Apr 21 \\
\hline 21 & -737 Aug 22 & -719 Sep 1 & -701 Sep 13 & -683 Sep 23 & -665 Oct 4 & -647 Oct 15 \\
\hline 22 & -736 Feb 15 & -718 Feb 26 & -700 Mar 8 & -682 Mar 19 & -664 Mar 29 & -646 Apr 10 \\
\hline 23 & -736 Aug 11 & -718 Aug 22 & -700 Sep 1 & -682 Sep 13 & -664 Sep 23 & -646 Oct 4 \\
\hline 24 & -735 Jan 5 & -717 Jan 16 & -699 Jan 27 & -681 Feb 7 & -663 Feb 17 & -645 Mar 1 \\
\hline 25 & -735 Jul 1 & -717 Jul 13 & -699 Jul 23 & -681 Aug 3 & -663 Aug 14 & -645 Aug 25 \\
\hline 26 & -735 Dec 25 & -716 Jan 6 & -698 Jan 16 & -680 Jan 28 & -662 Feb 7 & -644 Feb 18 \\
\hline 27 & -734 Jun 21 & -716 Jul 1 & -698 Jul 12 & -680 Jul 22 & -662 Aug 3 & -644 Aug 13 \\
\hline 28 & -734 Dec 15 & -716 Dec 25 & -697 Jan 6 & -679 Jan 16 & -661 Jan 28 & -643 Feb 7 \\
\hline 29 & -733 Jun 10 & -715 Jun 20 & -697 Jul 1 & -679 Jul 11 & -661 Jul 23 & -643 Aug 2 \\
\hline 30 & -733 Dec 5 & -715 Dec 15 & -697 Dec 26 & -678 Jan 6 & -660 Jan 17 & $-642 \operatorname{Jan} 27$ \\
\hline 31 & -732 Apr 30 & -714 May 11 & -696 Мау 21 & -678 Jun 2 & -660 Jun 12 & -642 Jun 23 \\
\hline 32 & -732 Oct 24 & -714 Nov 4 & -696 Nov 15 & -678 Nov 26 & -660 Dec 6 & -642 Dec 18 \\
\hline 33 & -731 Apr 19 & -713 May 1 & -695 May 11 & -677 Мay 22 & -659 Jun 2 & -641 Jun 13 \\
\hline 34 & -731 Oct 13 & -713 Oct 24 & -695 Nov 4 & -677 Nov 15 & -659 Nov 25 & -641 Dec 7 \\
\hline 35 & -730 Apr 9 & -712 Apr 19 & -694 May 1 & -676 May 11 & -658 May 22 & -640 Jun 2 \\
\hline 36 & -730 Oct 2 & -712 Oct 13 & -694 Oct 24 & -676 Nov 3 & -658 Nov 15 & -640 Nov 25 \\
\hline 37 & -729 Mar 30 & -711 Apr 9 & -693 Apr 20 & -675 Apr 30 & -657 May 12 & -639 May 22 \\
\hline 38 & -729 Sep 22 & -711 Oct 2 & -693 Oct 13 & -675 Oct 24 & -657 Nov 4 & -639 Nov 15 \\
\hline
\end{tabular}

number of days. ${ }^{6}$ In the mathematical lunar theory known as ACT System A (Neugebauer 1955 ) the length of the Saros assuming a constant solar velocity of $30^{\circ}$ per month is given by the so-called column $\Phi$. However, to obtain the true length of the Saros, a correction for solar anomaly would have to be added to $\Phi$, but such a function is so far unattested in the preserved texts. Thus, it would not be possible to use $\Phi$ to determine the time of predicted 
Table 1. Continued.

\begin{tabular}{|c|c|c|c|c|c|c|}
\hline & 7 & 8 & 9 & 10 & 11 & 12 \\
\hline 1 & -638 Apr 11 & -620 Apr 22 & -602 May 3 & -584 May 13 & -566 May 25 & -548 Jun 4 \\
\hline 2 & -638 Oct 6 & -620 Oct 16 & -602 Oct 26 & -584 Nov 7 & -566 Nov 18 & -548 Nov 29 \\
\hline 3 & -637 Apr 1 & -619 Apr 11 & -601 Apr 22 & -583 May 2 & -565 Мay 14 & -547 May 24 \\
\hline 4 & -637 Sep 25 & -619 Oct 6 & -601 Oct 17 & -583 Oct 27 & -565 Nov 8 & -547 Nov 18 \\
\hline 5 & $-636 \operatorname{Mar} 20$ & -618 Mar 31 & -600 Apr 11 & -582 Apr 22 & -564 May 2 & -546 May 14 \\
\hline 6 & -636 Sep 13 & -618 Sep 25 & -600 Oct 5 & -582 Oct 16 & -564 Oct 27 & -546 Nov 7 \\
\hline 7 & -635 Mar 10 & -617 Mar 21 & -599 Mar 31 & -581 Apr 12 & -563 Apr 22 & -545 May 3 \\
\hline 8 & $-635 \operatorname{Sep} 2$ & -617 Sep 14 & -599 Sep 24 & -581 Oct 5 & -563 Oct 16 & -545 Oct 27 \\
\hline 9 & -634 Jan 29 & -616 Feb 9 & -598 Feb 20 & -580 Mar 2 & -562 Mar 13 & -544 Mar 24 \\
\hline 10 & -634 Jul 24 & -616 Aug 3 & -598 Aug 15 & -580 Aug 25 & -562 Sep 5 & -544 Sep 16 \\
\hline 11 & -633 Jan 18 & -615 Jan 29 & -597 Feb 9 & -579 Feb 19 & -561 Mar 3 & -543 Mar 13 \\
\hline 12 & -633 Jul 14 & $-615 \mathrm{Jul} 24$ & -579 Aug 4 & -579 Aug 15 & -561 Aug 26 & -543 Sep 5 \\
\hline 13 & -632 Jan 7 & -614 Jan 18 & -596 Jan 29 & -578 Feb 8 & -560 Feb 20 & $-542 \operatorname{Mar} 2$ \\
\hline 14 & -632 Jul 2 & -614 Jul 14 & -595 Jul 24 & -578 Aug 4 & -560 Aug 15 & -542 Aug 26 \\
\hline 15 & -632 Dec 26 & $-613 \operatorname{Jan} 7$ & -595 Jan 17 & -577 Jan 28 & -559 Feb 8 & -541 Feb 19 \\
\hline 16 & -631 May 24 & 3 Jun 4 & -595 Jun & -577 Jun 25 & & -541 Jul 17 \\
\hline 17 & -631 Nov 16 & -613 Nov 27 & -595 Dec 8 & -577 Dec 19 & -559 Dec 29 & -540 Jan 10 \\
\hline 18 & -630 May 13 & -612 May 23 & -594 Jun 3 & -576 Jun 14 & -558 Jun 25 & $-540 \mathrm{Jul} 5$ \\
\hline 19 & -630 Nov 5 & -612 Nov 16 & -594 Nov 27 & -576 Dec 8 & -558 Dec 19 & -540 Dec 29 \\
\hline 20 & -629 May 2 & -611 May 12 & -593 Мау 23 & -575 Jun 3 & -557 Jun 14 & -539 Jun 24 \\
\hline 21 & -629 Oct 26 & -611 Nov 5 & -593 Nov 17 & -575 Nov 27 & -557 Dec 9 & -539 Dec 19 \\
\hline 22 & -628 Apr 20 & -610 May 1 & -592 May 12 & -574 May 23 & -556 Jun 2 & -538 Jun 13 \\
\hline 23 & -628 Oct 15 & -610 Oct 26 & -592 Nov 5 & -574 Nov 17 & -556 Nov 27 & -538 Dec 8 \\
\hline 24 & -627 Mar 11 & -609 Mar 22 & -591 Apr 2 & -573 Apr 13 & -555 Apr 23 & -537 May 5 \\
\hline 25 & -627 Sep 4 & -609 Sep 16 & -591 Sep 26 & -573 Oct 7 & -555 Oct 17 & -537 Oct 29 \\
\hline 26 & -626 Mar 1 & -608 Mar 11 & -590 Mar 22 & -572 Apr 2 & -554 Apr 13 & -536 Apr 23 \\
\hline 27 & -626 Aug 24 & -608 Sep 4 & -590 Sep 15 & -572 Sep 25 & -554 Oct 6 & -536 Oct 17 \\
\hline 28 & -625 Feb 18 & -607 Mar 1 & -589 Mar 12 & -571 Mar 22 & -553 Apr 3 & -535 Apr 13 \\
\hline 29 & -625 Aug 13 & -607 Aug 24 & -589 Sep 4 & -571 Sep 14 & -553 Sep 26 & -535 Oct 6 \\
\hline 30 & -624 Feb 8 & -606 Feb 18 & -588 Feb 29 & -570 Mar 12 & -552 Mar 22 & -534 Apr 2 \\
\hline 31 & $-624 \mathrm{Jul} 4$ & -606 Jul 15 & -588 Jul 25 & -570 Aug 5 & -552 Aug 16 & -534 Aug 27 \\
\hline 32 & -624 Dec 28 & -605 Jan 8 & -587 Jan 19 & -569 Jan 30 & -551 Feb 9 & -533 Feb 21 \\
\hline 33 & -623 Jun 23 & $-605 \mathrm{Jul} 5$ & -587 Jul 15 & -569 Jul 26 & -551 Aug 6 & -533 Aug 17 \\
\hline 34 & -623 Dec 17 & -605 Dec 28 & -586 Jan 8 & -568 Jan 19 & -550 Jan 29 & -532 Feb 10 \\
\hline 35 & -622 Jun 13 & -604 Jun 23 & -586 Jul 4 & -568 Jul 15 & $-550 \mathrm{Jul} 26$ & -532 Aug 5 \\
\hline 36 & -622 Dec 6 & -604 Dec 17 & -586 Dec 28 & -567 Jan 7 & -549 Jan 19 & -531 Jan 29 \\
\hline 37 & -621 Jun 2 & -603 Jun 13 & -585 Jun 24 & $-567 \mathrm{Jul} 4$ & -549 Jul 15 & -531 Jul 26 \\
\hline 38 & -621 Nov 26 & -603 Dec 6 & -585 Dec 18 & -567 Dec 28 & -548 Jan 8 & -530 Jan 19 \\
\hline
\end{tabular}

eclipses from earlier observations. In any case, eclipse times were being calculated well before it is believed $\Phi$ was probably constructed. We must therefore look elsewhere for what length of the Saros was used to predict eclipse times.

A clue to the answer to this question may come from some of the texts containing eclipse predictions themselves. The large compilation of lunar eclipse records mentioned above is 
Table 1. Continued.

\begin{tabular}{|c|c|c|c|c|c|c|}
\hline & 13 & 14 & 15 & 16 & 17 & 18 \\
\hline 1 & -530 Jun 15 & -512 Jun 25 & -494 Jul 7 & -476 Jul 17 & -458 Jul 28 & -440 Aug 7 \\
\hline 2 & -530 Dec 10 & -512 Dec 20 & -493 Jan 1 & -475 Jan 11 & -457 Jan 22 & -439 Feb 2 \\
\hline 3 & -529 Jun 4 & -511 Jun 15 & -493 Jun 26 & -475 Jul 6 & -457 Jul 17 & -439 Jul 28 \\
\hline 4 & -529 Nov 29 & -511 Dec 10 & -493 Dec 21 & -475 Dec 31 & -456 Jan 12 & -438 Jan 22 \\
\hline 5 & -528 May 24 & -510 Jun 4 & -492 Jun 14 & -474 Jun 26 & $-456 \mathrm{Jul} 6$ & -438 Jul 17 \\
\hline 6 & -528 Nov 17 & -510 Nov 29 & $-492 \operatorname{Dec} 9$ & -474 Dec 20 & -456 Dec 31 & -437 Jan 11 \\
\hline 7 & -527 May 14 & -509 May 25 & -491 Jun 4 & -473 Jun 15 & -455 Jun 26 & $-437 \mathrm{Jul} 7$ \\
\hline 8 & -527 Nov 6 & -509 Nov 18 & -491 Nov 28 & -473 Dec 9 & -455 Dec 20 & -437 Dec 31 \\
\hline 9 & -526 Apr 4 & -508 Apr 14 & -490 Apr 25 & -472 May 6 & -454 May 17 & -436 May 27 \\
\hline 10 & -526 Sep 27 & -508 Oct 7 & -490 Oct 19 & -472 Oct 29 & -454 Nov 9 & -436 Nov 20 \\
\hline 11 & -525 Mar 24 & -507 Apr 3 & -489 Apr 15 & -471 Apr 25 & -453 Мay 6 & -435 May 17 \\
\hline 12 & -525 Sep 17 & -507 Sep 27 & -489 Oct 8 & -471 Oct 19 & -453 Oct 30 & -435 Nov 9 \\
\hline 13 & -524 Mar 12 & -506 Mar 24 & -488 Apr 3 & -470 Apr 14 & -452 Apr 24 & -434 Мау 6 \\
\hline 14 & -524 Sep 5 & -506 Sep 17 & -488 Sep 27 & -470 Oct 8 & -452 Oct 19 & -434 Oct 30 \\
\hline 15 & -523 Mar 1 & -505 Mar 13 & -487 Mar 23 & -469 Apr 3 & -451 Apr 13 & -433 Apr 25 \\
\hline 16 & -523 Jul 27 & -505 Aug 8 & -487 Aug 18 & -469 Aug 29 & -451 Sep 9 & -433 Sep 20 \\
\hline 17 & $-522 \operatorname{Jan} 20$ & -504 Jan 31 & -486 Feb 11 & -468 Feb 22 & -450 Mar 4 & -432 Mar 15 \\
\hline 18 & -522 Jul 16 & -504 Jul 27 & -486 Aug 7 & -468 Aug 17 & -450 Aug 29 & -432 Sep 8 \\
\hline 19 & -521 Jan 10 & -503 Jan 20 & -485 Jan 31 & -467 Feb 11 & -449 Feb 22 & -431 Mar 4 \\
\hline 20 & -521 Jul 5 & -503 Jul 16 & -485 Jul 27 & -467 Aug 6 & -449 Aug 18 & -431 Aug 28 \\
\hline 21 & -521 Dec 30 & -502 Jan 10 & $-484 \operatorname{Jan} 21$ & -466 Jan 31 & -448 Feb 12 & -430 Feb 22 \\
\hline 22 & -520 Jun 24 & -502 Jul 5 & -484 Jul 15 & -466 Jul 27 & -448 Aug 6 & -430 Aug 17 \\
\hline 23 & -520 Dec 19 & $-502 \operatorname{Dec} 30$ & -483 Jan 10 & $-465 \operatorname{Jan} 21$ & -447 Jan 31 & -429 Feb 12 \\
\hline 24 & -519 May 15 & -501 May 26 & -483 Jun 5 & -465 Jun 17 & -447 Jun 27 & $-429 \mathrm{Jul} 8$ \\
\hline 25 & -519 Nov 8 & -501 Nov 19 & -483 Nov 30 & -465 Dec 11 & -447 Dec 22 & -428 Jan 2 \\
\hline 26 & -518 May 5 & -500 May 15 & -482 May 26 & -464 Jun 5 & -446 Jun 17 & -428 Jun 27 \\
\hline 27 & -518 Oct 28 & -500 Nov 7 & -482 Nov 19 & -464 Nov 29 & -446 Dec 11 & -428 Dec 21 \\
\hline 28 & -517 Apr 24 & -499 Мay 4 & -481 May 16 & -463 May 26 & -445 Jun 6 & -427 Jun 17 \\
\hline 29 & -517 Oct 17 & -499 Oct 28 & -481 Nov 8 & -463 Nov 18 & -445 Nov 30 & -427 Dec 10 \\
\hline 30 & -516 Apr 13 & -498 Apr 24 & -480 May 4 & -462 May 15 & -444 May 26 & -426 Jun 6 \\
\hline 31 & -516 Sep 7 & -498 Sep 18 & -480 Sep 28 & -462 Oct 10 & -444 Oct 20 & -426 Oct 31 \\
\hline 32 & -515 Mar 3 & -497 Mar 14 & -479 Mar 25 & -461 Apr 5 & -443 Apr 15 & -425 Apr 26 \\
\hline 33 & -515 Aug 27 & -497 Sep 8 & -479 Sep 18 & -461 Sep 29 & -443 Oct 10 & -425 Oct 21 \\
\hline 34 & -514 Feb 20 & -496 Mar 2 & -478 Mar 14 & -460 Mar 24 & -442 Apr 4 & -424 Apr 15 \\
\hline 35 & -514 Aug 17 & -496 Aug 27 & -478 Sep 7 & -460 Sep 18 & -442 Sep 29 & -424 Oct 9 \\
\hline 36 & -513 Feb 9 & -495 Feb 20 & -477 Mar 3 & -459 Mar 13 & -441 Mar 25 & -423 Apr 4 \\
\hline 37 & -513 Aug 8 & -495 Aug 16 & -477 Aug 28 & -459 Sep 7 & -441 Sep 18 & -423 Sep 28 \\
\hline 38 & $-512 \operatorname{Jan} 30$ & -494 Feb 9 & -476 Feb 21 & -458 Mar 3 & -440 Mar 13 & $-422 \operatorname{Mar} 25$ \\
\hline
\end{tabular}

known from three fragments. On only one of these (LBAT *1414) is the left edge preserved, and there we find the number 1,50 written next to the eclipse of 9 April $731 \mathrm{BC}$. This eclipse is the first eclipse in row 35 of the Saros scheme matrix. Unfortunately, since the remainder of the left edge is broken away, we cannot be sure if similar numbers were written next to each row of EPs, but it seems a reasonable hypothesis. Similar numbers, 
Table 1. Continued.

\begin{tabular}{|c|c|c|c|c|c|c|}
\hline & 19 & 20 & 21 & 22 & 23 & 24 \\
\hline 1 & -422 Aug 19 & -404 Aug 29 & -386 Sep 9 & -368 Sep 20 & -350 Oct 1 & -332 Oct 11 \\
\hline 2 & -421 Feb 13 & -403 Feb 23 & -385 Mar 7 & -367 Mar 17 & -349 Mar 28 & -331 Apr 8 \\
\hline 3 & -421 Aug 8 & -403 Aug 18 & -385 Aug 30 & -367 Sep 9 & -349 Sep 20 & -331 Oct 1 \\
\hline 4 & -420 Feb 2 & -402 Feb 13 & -384 Feb 24 & -366 Mar 6 & -348 Mar 17 & -330 Mar 28 \\
\hline 5 & -420 Jul 28 & -402 Aug 8 & -384 Aug 18 & -366 Aug 30 & -348 Sep 9 & -330 Sep 20 \\
\hline 6 & -419 Jan 21 & -401 Feb 2 & $-383 \mathrm{Feb} 12$ & -365 Feb 23 & -347 Mar 6 & -329 Mar 17 \\
\hline 7 & -419 Jul 17 & -401 Jul 29 & -383 Aug 8 & -365 Aug 19 & -347 Aug 30 & -329 Sep 10 \\
\hline 8 & $-418 \operatorname{Jan} 10$ & $-400 \operatorname{Jan} 22$ & -382 Feb 1 & -364 Feb 12 & -346 Feb 23 & -328 Mar 5 \\
\hline 9 & -418 Jun 8 & -400 Jun 18 & -382 Jun 29 & -364 Jul 9 & -3 & 31 \\
\hline 10 & -418 Dec 1 & -400 Dec 11 & -382 Dec 23 & -363 Jan 2 & -345 Jan 14 & -327 Jan 24 \\
\hline 11 & -417 May 28 & -399 Jun 7 & -381 Jun 18 & -363 Jun 29 & $-345 \mathrm{Jul} 10$ & -327 Jul 20 \\
\hline 12 & -417 Nov 21 & -399 Dec 1 & -381 Dec 12 & -363 Dec & -344 Jan 3 & -326 Jan 14 \\
\hline 13 & -416 May 16 & -398 May 27 & -380 Jun 6 & -362 Jun 18 & -344 Jun 28 & -326 Jul 9 \\
\hline 14 & -416 Nov 9 & -398 Nov 21 & -380 Dec 1 & -362 Dec 12 & -344 Dec 23 & -325 Jan 3 \\
\hline 15 & -415 May 5 & -397 May 16 & -379 May 17 & -361 Jun 7 & -343 Jun 17 & -325 Jun 28 \\
\hline 16 & & & & & & \\
\hline 17 & -414 Mar 26 & -396 Apr 5 & -378 Apr 17 & -360 Apr 27 & -342 May 8 & -324 May 19 \\
\hline 18 & -414 Sep 19 & -396 Sep 30 & -378 Oct 11 & & $-342 \mathrm{No}$ & -324 Nov 12 \\
\hline 19 & -413 Mar 16 & -395 Mar 26 & -377 Apr 6 & -359 Apr 17 & -341 Apr 28 & -323 May 8 \\
\hline 20 & -413 Sep 8 & -395 Sep 19 & -377 Sep 30 & -359 Oct 10 & -341 Oct 22 & -323 Nov 1 \\
\hline 21 & -412 Mar 4 & -394 Mar 16 & -376 Mar 26 & -358 Apr 6 & -340 Apr 17 & -322 Apr 28 \\
\hline 22 & -412 Aug 28 & $-394 \operatorname{Sep} 8$ & -376 Sep 18 & -358 Sep 30 & -340 Oct 10 & -322 Oct 21 \\
\hline 23 & $-411 \mathrm{Feb} 22$ & -393 Mar 5 & -375 Mar 15 & -357 Mar 27 & -339 Apr 6 & -321 Apr 17 \\
\hline 24 & $-411 \mathrm{Jul}$ & & $-3 / 5$ Ac & $-53 / \mathrm{Au}$ & $-339 \mathrm{Al}$ & -321 Sep 11 \\
\hline 25 & -410 Jan 12 & -392 Jan 23 & -374 Feb 3 & -356 Feb 14 & $-338 \mathrm{Feb}$ & -320 Mar 7 \\
\hline 26 & -410 Jul 8 & -392 Jul 19 & -374 Jul 30 & -356 Aug 9 & -338 Aug 21 & -320 Aug 31 \\
\hline 27 & -409 Jan 1 & -391 Jan 12 & -373 Jan 23 & -355 Feb 2 & -337 Feb 14 & -319 Feb 24 \\
\hline 28 & -409 Jun 28 & -391 Jul 8 & -373 Jul 20 & $-355 \mathrm{Jul} 30$ & -337 Aug 10 & -319 Aug 20 \\
\hline 29 & -409 Dec 22 & -390 Jan 1 & -372 Jan 12 & -354 Jan 23 & -336 Feb 3 & -318 Feb 13 \\
\hline 30 & -408 Jun 16 & -390 Jun 28 & -372 Jul 8 & -354 Jul 19 & -336 Jul 29 & -318 Aug 10 \\
\hline 31 & -408 Nov 11 & -390 Nov 22 & $-3 / 2 \mathrm{Dec} 2$ & -354 Dec 14 & -336 Dec 24 & -317 Jan 5 \\
\hline 32 & -407 May 7 & -389 May 18 & -371 May 28 & -353 Jun 9 & -335 Jun 19 & -317 Jun 30 \\
\hline 33 & -407 Oct 31 & -389 Nov 12 & -371 Nov 22 & -353 Dec 3 & -335 Dec 14 & -317 Dec 25 \\
\hline 34 & -406 Apr 26 & -388 May 6 & -370 May 17 & -352 May 28 & -334 Jun 8 & -316 Jun 18 \\
\hline 35 & -406 Oct 21 & -388 Oct 31 & -370 Nov 11 & -352 Nov 22 & -334 Dec 3 & -316 Dec 13 \\
\hline 36 & -405 Apr 15 & -387 Apr 26 & -369 May 7 & -351 May 17 & -333 May 29 & -315 Jun 8 \\
\hline 37 & -405 Oct 10 & -387 Oct 20 & -369 Oct 31 & -351 Nov 11 & -333 Nov 22 & -315 Dec 2 \\
\hline 38 & -404 Apr 4 & -386 Apr 15 & -368 Apr 26 & -350 May 7 & -332 May 17 & -314 May 29 \\
\hline
\end{tabular}

1,40 and 2,10, are found on the edge of LBAT 1413, next to the records of eclipses on 6 February $747 \mathrm{BC}$ and 2 August $747 \mathrm{BC}$, which correspond to the first eclipses in rows 1 and 2 of the matrix. For want of a better term, we will call these 'strange numbers'. As first recognized by Brown (2000, p. 205) and then independently by us, ${ }^{7}$ if these 'strange numbers' are taken to be in $u \check{s}$, then they are approximately of the right size to represent 
the length of the Saros. Our interpretation differs somewhat in other details from Brown's, however.

Pinches' copy of LBAT 1413, from which Brown is presumably working, does not show the number 2,10 at the beginning of the third line (the number has only become visible after recent cleaning). Brown writes that the numbers 'appear to pertain to the year in question', but the presence of the newly visible number 2,10 indicates that the numbers are not connected to the whole year but instead to the eclipses, as is to be expected, since the Saros length varies greatly over the course of the year. Furthermore, we believe that the number 1,50 written on the side of the large compilation was intended to apply to all eclipses in that row of the matrix, not just the first eclipse. Quite probably, the numbers on LBAT 1413 were taken from a similar source and added to the eclipse records on LBAT 1413 but originally meant to refer to all eclipses in their rows of the matrix. There are, we believe, several pieces of evidence in support of this view.

First of all, if the numbers were used, as Brown tentatively suggests, to calculate the times of the eclipses of 6 February 747 BC, 2 August 747 BC, and 9 April 731 BC by adding them to the times of observed eclipses 1 Saros earlier, we would have to assume that regular eclipse observations were being recorded at least 1 Saros before $747 \mathrm{BC}$. No such observations are preserved, and there is a small amount of circumstantial evidence that eclipse records were only kept regularly from $747 \mathrm{BC}{ }^{8}$ Furthermore, if the numbers 1,40 and 2,10 are contemporary with the eclipses in $747 \mathrm{BC}$, it is strange that - so far as is preserved-the times of these eclipses are given only very roughly by the watch. The numbers would seem to be redundant. Finally, it may be worthy of note that the numbers actually agree best with computed values of the length of the Saros somewhat later than ca $750 \mathrm{BC}$ (see below). None of these arguments are conclusive, but together they do suggest that we should not associate the numbers only with the eclipses of 6 February $747 \mathrm{BC}$, 2 August $747 \mathrm{BC}$, and 9 April $731 \mathrm{BC}$ but instead with all of the eclipses in those Saros series. Consequently, we do not believe it likely that the numbers were used-or indeed available to use-in the mid-eighth-century BC, nor that they come directly from observation of any particular eclipse pair. Instead, we believe that the numbers come from some function that models the length of the Saros. Below we discuss two such functions, one that gives the length of the Saros at 1-year intervals and the other that models the change in the Saros length during a year.

\section{A Simple 18-Year Function for the Length of the Saros}

BM 45861 is a fragment of an astronomical procedure text from Babylon. ${ }^{9}$ Of the remaining text, only one section is sufficiently well preserved to be interpreted. This section contains numbers that form a linear zigzag function, with maximum 2,20 uš, minimum $1,35 u \check{s}$, difference $5 u \check{s}$, and period 18. Following each number is the word tardītu, a noun meaning something like 'addition' or 'extra', an apt description since it is very likely that 
the numbers relate to the additional length of time over a whole number $(6,585)$ of days that make up a Saros period of 223 synodic months. Two hundred and twenty-three synodic months last about 10 days over 18 years, and so our text apparently gives a function for the length of the Saros at yearly intervals. We present the function below in schematic format (Table 2). ${ }^{10}$

Table 2. The BM 45861 'Saros length' zigzag function.

\begin{tabular}{llll}
\hline E1 & 1,35 & subtract & 5 \\
E2 & 1,40 & add & 5 \\
E3 & 1,45 & add & 5 \\
E4 & 1,50 & add & 5 \\
E5 & 1,55 & add & 5 \\
E6 & 2,0 & add & 5 \\
E7 & 2,5 & add & 5 \\
E8 & 2,10 & add & 5 \\
E9 & 2,15 & add & 5 \\
E10 & 2,20 & add & 5 \\
E11 & 2,15 & subtract & 5 \\
E12 & 2,10 & subtract & 5 \\
E13 & 2,5 & subtract & 5 \\
E14 & 2,0 & subtract & 5 \\
E15 & 1,55 & subtract & 5 \\
E16 & 1,50 & subtract & 5 \\
E17 & 1,45 & subtract & 5 \\
E18 & 1,40 & subtract & 5 \\
E19 & 1,35 & subtract & 5 \\
\hline
\end{tabular}

The zigzag function found on BM 45861, which we shall henceforth call the 'simple 18-year function', could presumably have been used to predict the times of future eclipses. Steele (2002) discusses how the function may have been derived from observations, and there is no need to repeat all of the details here. A brief outline may prove useful, however.

The construction of the function probably came in two steps. From basic timed observations of eclipses, it could be noted that the length of the Saros varies between roughly 1,35 and $2,15 u \check{s}$, but that it is roughly constant within a Saros series. Thus, the variation in the length of the Saros is roughly periodic, with the Saros itself as the period. It could also be noted that at successive EPs separated by 6 or 5 months, the Saros length is inverted, i.e. if it is large at one EP, it is small at the next, etc. However, for eclipses separated by two EPs (i.e. at 12 or 11 months), the Saros length changes by about 5 $u \check{s}$ up or down. ${ }^{11}$ This suggests that the 38 EPs within a Saros be divided into two sequences, the odd-numbered eclipses in the Saros scheme matrix and the even-numbered eclipses. In fact, these two sequences correspond to the two nodes, ascending or descending, near which the eclipse takes place. Both this and the inverse behavior of the Saros length provide a motivation for treating the odd and even sequences separately. Once this 


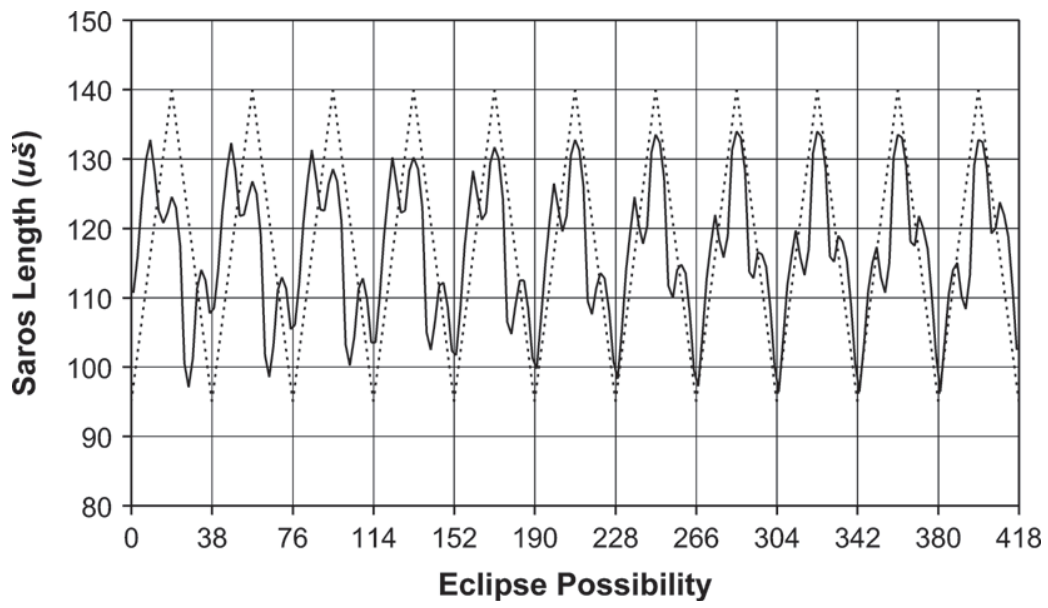

Fig. 1. The variation of the Saros length for odd-numbered eclipse possibilities beginning with the eclipse of 6 February 747 BC. The plain line gives the Saros lengths associated with the odd-numbered eclipses, and the dashed line is the 18-year zigzag function.

split into odd- and even-numbered eclipses had been made, fitting a linear zigzag function to the observed Saros lengths is quite a plausible next step and one that works very well. ${ }^{12}$ However, fitting a zigzag function directly to the 19 eclipses in either the odd or even sequences is complicated by the fact that 19 is not a nice number, and one cannot obtain integer numbers for the amplitude and difference of a zigzag function with 19 as its period. Nineteen EPs correspond to 1 Saros, or just over 18 years, and 18 does lead to nice numbers for the zigzag function. Indeed, it leads to the parameters of the zigzag function found on BM 45861, suggesting that this may well have been how the function was constructed.

Let us now look at how this simple 18-year function compares with nature. In Figure 1, we plot the variation in the Saros length (the excess over 6,585 whole days of the time interval between this eclipse and that 223 months later) as given by modern computation and by this simple 18-year function for all of the odd-numbered eclipses over 11 SCs. ${ }^{13}$ For our present purposes, we have chosen that this first eclipse is at a minimum of the function, since then we have fairly good agreement between the function and nature.

First, we can see that although the maximum value of the simple 18-year function is approximately $5 u \check{s}$ greater than the true maximum length of the Saros, the simple zigzag function is nevertheless a good approximation to nature. Most importantly, the period of the function is fairly good, so it continues to be a realistic representation of nature after several SCs. In particular, it is clear from the plot that even after 11 SCs (ca 200 years), the function is still reasonably in phase with nature and could certainly be used with moderate effectiveness. However, it is possible that if the function was used over 


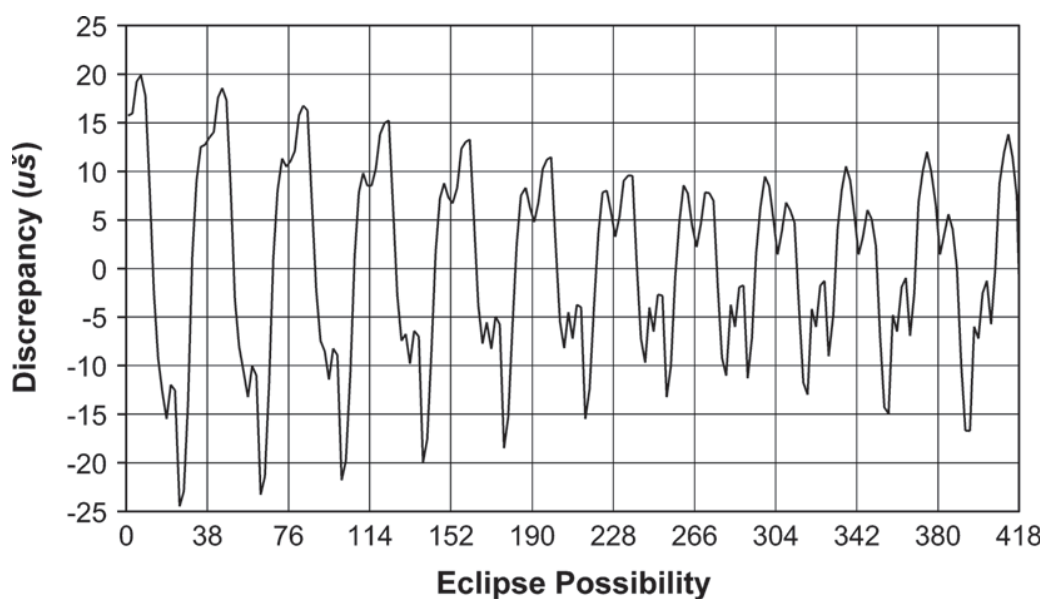

Fig. 2. The discrepancy between the 18-year zigzag function and computed length of the Saros.

this length of time, the discrepancy between the function and nature would have been noted.

Figure 2 shows the difference between the simple 18-year function and nature. In constructing this plot, we have modified the function slightly in order to compare the 18 values from the zigzag function directly with the 19 values for each eclipse number within an SC. The modification adopted was simply to repeat the minimum value at both the final two eclipse numbers within each Saros. Since for the moment we are only interested in making a general comparison between the simple 18-year function and nature, this modification does not have any appreciable effect.

It is immediately clear that the accuracy of the function is not constant over time. At the point of 'optimal fit' (arbitrarily placed around EP number 266 for the sake of our discussion), the discrepancy between the zigzag function and nature varies between about +10 and $-10 u \check{s}$. This discrepancy is caused purely by the use of a simple zigzag function to model a much more complicated function. Nevertheless, it is worth remarking that a range of discrepancies from +10 to $-10 u \check{s}$ is not at all bad, especially when one considers that the zigzag function is only given in units of $5 u \check{s}$. Furthermore, the early eclipse records (before ca $570 \mathrm{BC}$ ) are themselves recorded with a precision of only $5 u \check{s}$ (and were timed with an accuracy several times worse than that), ${ }^{14}$ so the observed difference between the zigzag function and nature at this period of 'optimal fit' may have been somewhat less than $\pm 10 u \check{s}$, although importantly the fact that there was a discrepancy probably could be seen.

Over longer time periods, however, we can see that the amplitude of the discrepancy increases. Six SCs earlier than the 'optimal fit', the discrepancy range is approximately 
double that at the time of 'optimal fit'. This change in the amplitude of the discrepancy is caused by the fact the Saros relation is not perfect. In particular, the Saros is approximately 10 days longer than 18 solar years, and so we are about $10 / 360=1 / 36$ further advanced in the cycle of solar anomaly. It will, therefore, take 36 Saroi, roughly 648 years, before we will once more be at the same solar anomaly, and the zigzag function will once more be in good agreement with nature. There is also a slight effect due to the fact that 1 Saros of 223 synodic months does not equate precisely to 239 anomalistic months but is 0.2 days shorter. Thus, there is a small change in lunar anomaly after 1 Saros. We are only about $0.2 / 27.5$ days different in the cycle of lunar anomaly, however, and this will therefore have a much smaller effect than the change in solar anomaly after 1 Saros. The long-term discrepancies are therefore due primarily to solar anomaly.

Looking more closely at the discrepancies between the function and nature, we see that there is also some small-scale structure. Within each Saros of the discrepancies, there are two peaks and two troughs. There is no fixed relation between the double peaks, however. Sometimes the larger is first, sometimes second. The most likely explanation for this is that the discrepancy is caused by two factors: one solar anomaly and the other lunar anomaly. The simple zigzag function gives 18 values for the length of the Saros over the course of an SC. We postulate below that the 18 values of the zigzag function were applied to the 19 even or odd EPs by repeating either the maximum or minimum value. In general, therefore, successive values of the zigzag function were associated with eclipses at 12-month intervals, which corresponds to about 10 or 11 days short of a solar year. Therefore, there is a noticeable change in solar anomaly for each value. However, 12 synodic months are also about 4 days shorter than 13 anomalistic months, so there is an additional contribution to the change in the length of the Saros due to the change in lunar anomaly. However, the changes in lunar and solar anomaly after 12 synodic months do not have the same period. It will take 36 sets of 12 synodic months before the solar anomaly is roughly the same but only 14 sets of 12 synodic months before the lunar anomaly is roughly the same.

It is very unlikely that the Babylonian astronomers could have got any information out of the discrepancies between the zigzag function and observation, other than that the discrepancy is based upon different factors. It would not be a huge step to recognize that two main factors were connected with the sun and moon, but no quantitative information could be gained. Nevertheless, it seems quite possible that this and similar functions such as the one discussed below provided the idea-or at least contributed towards formulating the idea - that it was necessary to separate the Saros length into solar and lunar components. We see a recognition of the inadequacies of the simple 18-year function as a possible source of the realization that it was necessary to study the movement of the sun and moon in detail in order to obtain a method for determining an accurate Saros length.

Before going any further, it is worth investigating another cuneiform text TU 11 (AO 6455), which gives rules for finding the shift in time between eclipses situated 1 Saros apart. 


\section{The TU 11 Method for Predicting Eclipses}

In sections 9-12 of TU 11 (see Brack-Bernsen and Hunger 2002, pp. 80-85), a method for predicting the time of an eclipse was introduced through four examples. Each example starts with the time $t_{0}$ of a known eclipse (possibility) and ends with the expected time $t$ of the eclipse which was to occur 1 Saros later.

One way of interpreting the procedure, and the interpretation which we think of as the most probable, is the following: the shift in time between two lunar eclipses, situated 1 Saros apart, was taken to be an entire number of days plus 1 mina $+1 / 3 \times$ the length of the night. For each month, the night's length could be found from the scheme of daylength in MUL.APIN ${ }^{15}$ or in Enuma Anu Enlil (EAE) XIV. ${ }^{16}$ We render the procedure for finding $t$, the time of the new eclipse, in a formula:

$$
t=t_{0}+1 / 3 \text { night }+1
$$

The time $t_{0}$ of the 'old eclipse' was given by its distance in time to the nearest sunset or sunrise. Knowing the length of day and night, the procedure could predict the time of a coming eclipse.

Let us look a little closer on the function $t-t_{0}=(1 / 3$ night +1$)$ for the shift in time between the two eclipses situated 1 Saros apart. According to the schemes in MUL.APIN or in EAE XIV, the length of the night was a function of the month - a linear zigzag function-with the period of a solar year. Consequently, the time intervals between eclipses, $t-t_{0}$, found according to the procedure, will also be situated on a linear zigzag function with the period of the solar year, if the values are tabulated at consecutive months, as we have done in column 4 of Table 3. If, however, as in our case, the timeshift (between eclipse lunations situated 1 Saros apart) is only considered and tabulated at intervals of 12 or 11 months, then the TU 11 procedure leads to a numerical fit, which could be described as a linear zigzag function interrupted by small steps. See Figure 3 which compares the actual Saros shift (measured in units of $u \check{s}$ ) to the shift computed according to the TU 11 procedure. In the figure, we have plotted the Saros shift for all odd eclipse lunations including those where a lunar eclipse was not visible in Mesopotamia. We do it, knowing that for most eclipse lunations the Saros length can be found by observed eclipses situated 1, 2, or 3 Saroi apart.

We see this excellent fit as a confirmation of the proposed interpretation of section 9 of TU 11 that the shift in time between lunar eclipses 18 years apart was calculated as $(1 / 3$ night +1$)$. It follows that the length of the Saros would be 6,585 days + $(1 / 3$ night +1$)$.

In the first part of this paper, we presented a linear zigzag function for the length of the Saros, tabulated at yearly intervals over a period of 18 years. Inspired by this function, we shall now similarly, for yearly intervals, construct a linear zigzag function for the length of the Saros based on the TU 11 method. It means that we will smoothen out the 'thick curve' in figure 3. In Table 3, we have constructed the numerical values of the TU 11 
Table 3. The Saros length according to the TU 11 procedure.

\begin{tabular}{cccc}
\hline $\begin{array}{c}\text { Length of night } \\
\text { (mina) }\end{array}$ & $\begin{array}{c}\text { EAE XIV } \\
\text { month }\end{array}$ & $\begin{array}{c}\text { MUL.APIN } \\
\text { month }\end{array}$ & $\begin{array}{c}t-t_{0}=1 / 3 \text { night }+1 \\
\text { (mina) }\end{array}$ \\
\hline 4 & IX & X & $2 ; 20,00$ \\
$3 ; 40$ & X & XI & $2 ; 13,20$ \\
$3 ; 20$ & XI & XII & $2 ; 06,40$ \\
3 & XII & I & $2 ; 00,00$ \\
$2 ; 40$ & I & II & $1 ; 53,20$ \\
$2 ; 20$ & II & III & $1 ; 46,40$ \\
2 & III & IV & $1 ; 40,00$ \\
$2 ; 20$ & IV & V & $1 ; 46,40$ \\
$2 ; 40$ & V & VI & $1 ; 53,20$ \\
3 & VI & VII & $2 ; 00,00$ \\
$3 ; 20$ & VII & VIII & $2 ; 06,40$ \\
$3 ; 40$ & VIII & IX & $2 ; 13,20$ \\
4 & IX & X & $2 ; 20,00$ \\
\hline
\end{tabular}

function. According to the MUL.APIN daylength scheme, the equinoxes (at which day $=$ night $=3$ minas) are assumed to take place on the 15 th of months I and VII, while the shortest night ( $=2$ minas) is that of the 15th of month IV and the longest night $(=4$ minas) occurs on the 15th of month X. EAE XIV table C gives the same values for day and night; however, the corresponding dates are shifted 1 month backwards. According to the EAE conventions, equinox is put on the 15th of months XII and VI. In Table 3, column 1 gives the schematic daylengths measured in mina and columns 2 and 3 list the corresponding month according to the EAE and MUL.APIN conventions, respectively. Column 4 lists the shift in time $t-t_{0}$ between eclipses, calculated from the length of night given in column 1.

The times are here given in mina, but they can be converted to units of $u \check{s}$ (time-degrees) by multiplying the numbers by 60 , i.e. by shifting the ';' one place to the right, according to the well-known relation: 1 mina $=60 u \check{s}=1,00 u \check{s}$. TU 11 says nothing about the units used. Perhaps, the numbers were meant to be interpreted both as $u \check{s}$ and as mina.

Can this function eventually give the 'strange numbers' from the large SC table? Only if we use rounded numbers because in Table 4, we do not find the 'strange numbers' 1,50 or 2,10. The usual Babylonian way of rounding is to throw away unneeded digits. If the numbers found by the TU 11 procedure are rounded to $10 u \check{s}$, then 2,13,20 would become 2,10, the number 1,53,20 would become 1,50, and 1,46,40 would become 1,40.

We return to the SC scheme. Knowing the Babylonian months numbers of the eclipse dates, we have tested if there are columns in the SC scheme where the TU 11 procedure, applied to the months in lines 1,2, and 35 and then rounded, will deliver the numbers 1,40, 2,10 , and 1,50 . We found only two fits. The first fit is found in cycle 12 (starting 4 June 549 $\mathrm{BC}$ ). Here, we get the numbers searched for, if we determine the length of night according to the EAE scheme. The second fit is found in cycle 15 (starting 7 July $495 \mathrm{BC}$ ), if we use the night lengths from the MUL.APIN scheme. 


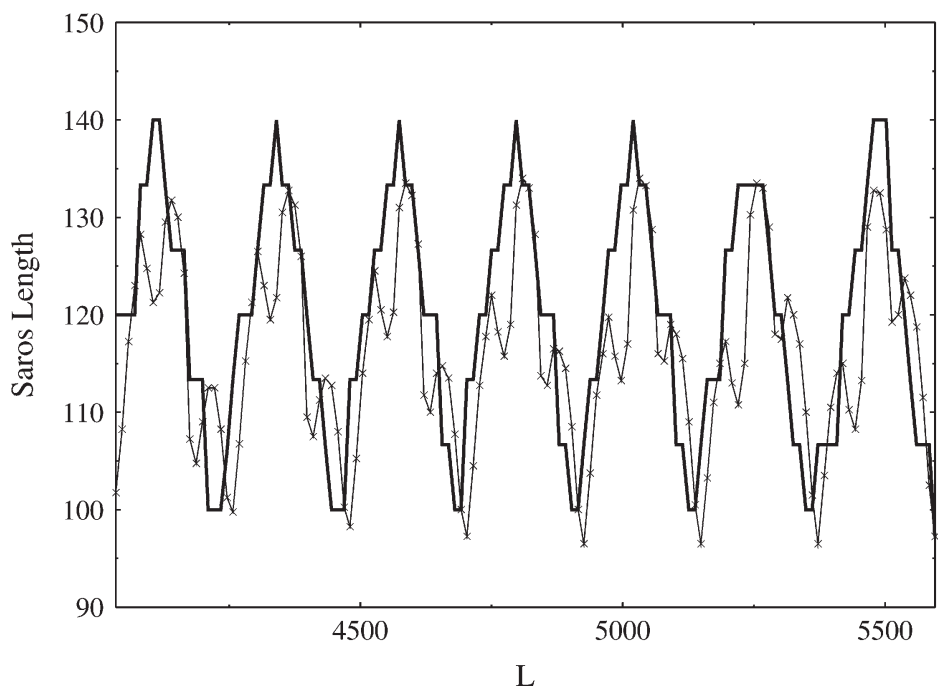

Fig. 3. Checking the accuracy of the TU 11 procedure. For a series of 'odd' eclipse possibilities (EPs) from the Saros cycle (SC) scheme, the actual Saros length is compared to the values found according to the TU 11 procedure. The comparison takes place over 7 consecutive Saroi, starting with the first EP of SC 5, 21 March 675 BC, and ending with EP 1 of SC 12, 4 June 549 BC. As independent variable, we use $L$, i.e. the Goldstine number for the respective lunations. The Saros lengths, measured from consecutive EPs at descending node, are marked by crosses and connected by a thin line. The TU 11 function is drawn with a thick line.

It is therefore possible, but by no means proven, that the numbers found on LBAT 1413, etc. are calculated as described above, although we have no explanation for why cycles 12 or 15 would have been chosen to give the numbers. Nevertheless, the existence of the TU 11 procedure shows us that the Babylonians had numerical models for calculating the lengths of the Saros by a method which can be summarized as a zigzag function with the period of 1 year. Is there also a connection between this function and the 'simple 18-year function' presented above?

\section{The TU 11 Predicting Method and the SC Scheme}

Now we shall demonstrate how a linear zigzag function with the period of 18 years can be derived from the the TU 11 rule. In so doing, we shall just combine the SC scheme with the TU 11 predicting method, refining ourselves only to those practices known from early Babylonian astronomy which, for example, can be found in MUL.APIN.

We consider a column, say column 8 , in the SC scheme, i.e. those 38 lunations within the 18 years from $621 \mathrm{BC}$ to $604 \mathrm{BC}$, at which a lunar eclipse could possibly take place. Suppose that the time of these eclipses or EPs is known. We shall now show what comes out if the shifts in time to the eclipses (or possibilities) in the next column, i.e. in column 
Table 4. For eclipse possibilities EP 1 to EP 38 from Sarcos cycle (SC) 8, we list the Babylonian months together with the Saros length as given by the TU 11 method. The data for the 19 odd-numbered EPs (near descending node) are listed in columns 2 and 3, while those for the 19 EPs around ascending node are listed in columns 4 and 5.

\begin{tabular}{|c|c|c|c|c|}
\hline \multirow[t]{2}{*}{ SC 8} & \multicolumn{2}{|c|}{ EPs at descending node } & \multicolumn{2}{|c|}{ EPs at ascending node } \\
\hline & Month & TU 11 timeshift & Month & TU 11 timeshift \\
\hline EP 1 & II & 1,$46 ; 40$ & & \\
\hline EP 2 & & & VIII & 2,$13 ; 20$ \\
\hline EP 3 & I & 1,$53 ; 20$ & & \\
\hline EP 4 & & & VII & 2,$06 ; 40$ \\
\hline EP 5 & I & 1,$53 ; 20$ & & \\
\hline EP 6 & & & VII & 2,$06 ; 40$ \\
\hline EP 7 & $\mathrm{XII}_{2}$ & 2,$00 ; 00$ & & \\
\hline EP 8 & & & VI & 2,$00 ; 00$ \\
\hline EP 9 & XI & 2,$06 ; 40$ & & \\
\hline EP 10 & & & V & 1,$53 ; 20$ \\
\hline EP 11 & XI & 2,$13 ; 20$ & & \\
\hline EP 12 & & & V & 1,$46 ; 40$ \\
\hline EP 13 & X & 2,$13 ; 20$ & & \\
\hline EP 14 & & & IV & 1,$46 ; 40$ \\
\hline EP 15 & $X$ & 2,$13 ; 20$ & & \\
\hline EP 16 & & & III & 1,$40 ; 00$ \\
\hline EP 17 & IX & 2,$20 ; 00$ & & \\
\hline EP 18 & & & II & 1,$46 ; 40$ \\
\hline EP 19 & VIII & 2,$13 ; 20$ & & \\
\hline EP 20 & & & II & 1,$46 ; 40$ \\
\hline EP 21 & VIII & 2,$13 ; 20$ & & \\
\hline EP 22 & & & II & 1,$46 ; 40$ \\
\hline EP 23 & VII & 2,$06 ; 40$ & & \\
\hline EP 24 & & & XII & 2,$00 ; 00$ \\
\hline EP 25 & VI & 2,$00 ; 00$ & & \\
\hline EP 26 & & & XII & 2,$00 ; 00$ \\
\hline EP 27 & VI & 2,$00 ; 00$ & & \\
\hline EP 28 & & & XII & 2,$00 ; 00$ \\
\hline EP 29 & VI & 2,$00 ; 00$ & & \\
\hline EP 30 & & & XII & 2,$00 ; 00$ \\
\hline EP 31 & V & 1,$53 ; 20$ & & \\
\hline EP 32 & & & $\mathrm{X}$ & 2,$13 ; 20$ \\
\hline EP 33 & IV & 1,$46 ; 40$ & & \\
\hline EP 34 & & & $\mathrm{X}$ & 2,$13 ; 20$ \\
\hline EP 35 & III & 1,$40 ; 00$ & & \\
\hline EP 36 & & & IX & 2,$20 ; 0$ \\
\hline EP 37 & III & 1,$40 ; 00$ & & \\
\hline EP 38 & & & IX & 2,$20 ; 0$ \\
\hline
\end{tabular}


9, 1 Saros later are calculated by means of the formula given above and smoothened out to give a linear zigzag function.

Inspired by the Babylonians, we shall also divide the 38 eclipse possibilities into two sequences, each containing 19 EPs which take place at intervals of 12 or 11 synodic months. We are justified in doing this, by examples such as Text $S$ (a text concerned with solar eclipses), ${ }^{17}$ where the two sequences of 19 EPs in several respects were treated as separate sequences. Column III of Text $\mathrm{S}$, which gives the values of a function associated with the eclipse magnitude (of solar eclipses), is calculated separately for each of the two sequences of 19 conjunctions: those 19 taking place near the ascending node and those 19 taking place near the descending node.

In Table 4, we list the Babylonian month of the EPs from SC 8, together with the timeshift calculated by the TU 11 method and using the date convention according to the schemes of EAE. (Had we used another column or the MUL.APIN date convention, we would have found very similar number columns of the same structure. The reason for choosing the EAE convention is that the intercalation of a 13th month undertaken by the Babylonians for the period of interest, SC 8-SC 17, let vernal equinox fall in month XII. This is in agreement with the EAE convention.) In the left columns, we list months and timeshift of those eclipse dates which occur around the descending node, while the data from the EPs around ascending node are listed in the right two columns.

It is immediately seen that the numbers listed in columns 3 and 5, although sometimes repeating the same number, increase and decrease in such a way that they can be fitted nicely with a linear zigzag function. The maximum value is 2,20;00, while minimum is 1,40;00, the amplitude being 40;00 ǔs. Each column has 19 values; column 3, e.g. starts with 1,$46 ; 40$, increasing until 2,20;00 and then decreasing to 1,$40 ; 00$. However, the number of lines, 19, is an odd number which does not divide into the variation of $80=2 \times$ the difference 40. An obvious solution to this problem would be to use 18 steps and to repeat one value. Either the maximum value could be taken twice or the minimum value could be taken twice. Then, one would have the situation where a change of 40;00 (from maximum to minimum or vice versa) takes place over nine lines, so that the change over one line would be $4 ; 26,40=40 \div 9$. To give an example, we shall concentrate on the numbers in column 3 and show how this number column can be fitted by a linear zigzag function. We give two possible solutions.

In Table 5, we have let the zigzag function have nine different values on each branch, repeating one number, either the maximum value of 2,20;00,00 or starting and ending with the minimum value. In this way, we end up with a nice symmetric function. The same zigzag functions can be used for fitting the numbers in column 5 of Table 4-we just have to start with a suitable value, i.e. to shift the function by 9 or 10 lines.

Figure 4 shows that this numerical function fits the empirical data, i.e. the real differences in time between eclipses 1 Saros apart, quite well-as well as was the case for the simple 18-year function (compare with Figure 1). 
Table 5. Best fitting zigzag functions.

\begin{tabular}{rrr}
\hline Eclipse - Number & Minimum twice & Maximum repeated \\
\hline EP 1 $=$ E1 & 1,$40 ; 00,00$ & 1,$44 ; 26,40$ \\
EP 3 $=$ E2 & 1,$44 ; 26,40$ & 1,$48 ; 53,20$ \\
EP 5 $=$ E3 & 1,$48 ; 53,20$ & 1,$53 ; 20,00$ \\
EP 7 $=$ E4 & 1,$53 ; 20,00$ & 1,$57 ; 46,40$ \\
EP 9 $=$ E5 & 1,$57 ; 46,40$ & 2,$02 ; 13,20$ \\
EP 11 $=$ E6 & 2,$02 ; 13,20$ & 2,$06 ; 40,00$ \\
EP 13 $=$ E7 & 2,$06 ; 40,00$ & 2,$11 ; 06,40$ \\
EP 15 $=$ E8 & 2,$11 ; 06,40$ & 2,$15 ; 33,20$ \\
EP 17 $=$ E9 & 2,$15 ; 33,20$ & 2,$20 ; 00,00$ \\
EP 19 $=$ E10 & 2,$20 ; 00,00$ & 2,$20 ; 00,00$ \\
EP 21 $=$ E11 & 2,$15 ; 33,20$ & 2,$15 ; 33,20$ \\
EP 23 $=$ E12 & 2,$11 ; 06,40$ & 2,$11 ; 06,40$ \\
EP 25 $=$ E13 & 2,$06 ; 40,00$ & 2,$06 ; 40,00$ \\
EP 27 $=$ E14 & 2,$02 ; 13,20$ & 2,$02 ; 13,20$ \\
EP 29 $=$ E15 & 1,$57 ; 46,40$ & 1,$57 ; 46,40$ \\
EP 31 $=$ E16 & 1,$53 ; 20,00$ & 1,$53 ; 20,00$ \\
EP 33 $=$ E17 & 1,$48 ; 53,20$ & 1,$48 ; 53,20$ \\
EP 35 $=$ E18 & 1,$44 ; 26,40$ & 1,$44 ; 26,40$ \\
EP 37 $=$ E19 & 1,$40 ; 00,00$ & 1,$40 ; 00,00$ \\
EP 1 $=$ E20 & 1,$40 ; 00,00$ & 1,$40 ; 00,00$ \\
\hline
\end{tabular}

For comparison, in Table 6 we render the reconstructed zigzag function, in a similar way as the 'simple 18-year function' was presented on BM 45861.

The zigzag function proposed here has 19 values, starting and ending with the minimal value of $1,40 u \check{s}$. (We could as well have repeated the maximum value of 2,20ǔs.) We do this in order to get a symmetric function. Another solution would be just to skip one value and give only 18 values, one for each year of the Saros, as is the case for BM 45861.

The zigzag function in Table 5 above was constructed like many zigzag functions known: starting with the total variation of a quantity, i.e. the maximum value $M$ and its minimum value $m$, and finding intermediate values by linear interpolation. As textual evidence of this practice, the daylength schemes from MUL.APIN can be put forward. The so-called first intercalation scheme (tablet II i 9-24) gives the ideal dates of summer solstice, fall equinox, and winter solstice, together with the length of day and night: the shortest night lasts 2 minas; the equinoctal night, 3 minas; and the longest night, 4 minas. In the so-called Waterclock (tablet II ii 43-iii 15), ${ }^{18}$ the length of night is listed for many more dates, the 1 st and 15th days of all 12 months of the ideal year. The numerical values for the duration of night on these dates form a linear zigzag function, with minimum 2 and maximum 4. Thus, we can conclude that they are found through linear interpolation between the extreme values of 2 and 4 minas for the shortest and longest night.

We stress again that for the hypothetical reconstruction of the Saros length zigzag function, we have only used the practices known from early Babylonian astronomy in 


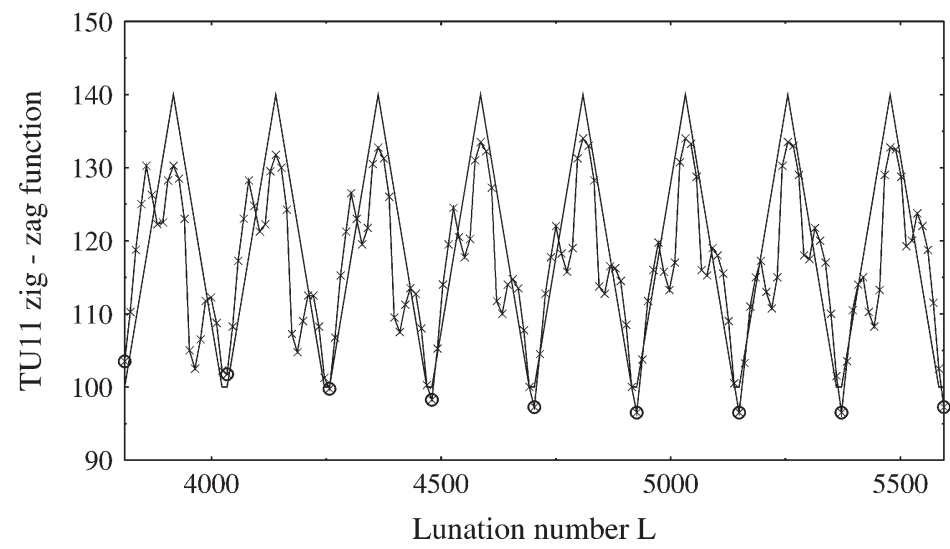

Fig. 4. For a series of 'odd' eclipse possibilities (EPs) from the Saros cycle (SC) scheme, the Saros length is compared to the reconstructed TU 11 Saros length zigzag function. The comparison takes place over 8 consecutive Saroi, starting with the very first EP of SC 4, 10 March 693 BC, and ending with EP 1 of SC 12 , 4 June 549 BC. The first EP of each SC is marked by a circle. As independent variable, we use $L$, i.e. the Goldstine number for the respective lunations. The Saros length, measured from consecutive EPs at descending node, is marked by crosses and connected by a thin line. The TU 11 zigzag function is drawn with a thick line.

connection with the predicting method from TU 11 and the daylength schemes from EAE or MUL.APIN. In early Babylonian astronomy, it was a general approach to derive astronomical quantities from the day or night length. ${ }^{19}$ Many rules on TU 11 provide further examples of how the length of day or night was used as a generating function for other astronomical quantities. ${ }^{20}$

The 'simple 18-year function' is very similar to the reconstructed TU 11 Saros length function. The question arises: are they somehow connected or derived independently of each other. It is possible that the '18-year function' is just a rounded version of the TU 11 function: using the more practical difference of $5 u \check{s}$ per year (or line) instead of 4;26,40 will result in an amplitude of $9 \times 5=45$ instead of 40 . This can, for example, be obtained by changing the minimum from 1,40 to 1,35 . Another possibility would be that the 'simple 18-year function' was derived empirically, i.e. directly from observed times of eclipses 1 Saros apart. Studying the SC scheme, a connection between the actual month (which is linked to length of night) and Saros duration can have been found (later) and utilized to formulate the predicting rules for eclipses on TU 11. A third possibility is that the two functions are constructed independently of each other. Unfortunately, we lack the evidence to decide between these possibilities.

Aside from the question of whether the TU 11 function and the 'simple 18-year function' are related, we now have evidence that the solar contribution of the duration of the Saros was treated numerically and theoretically within early Babylonian astronomy. In the 'simple 18-year function', we find a numerical sequence which fits nature quite well 
Table 6. The reconstructed TU 11 'Saros length' zigzag function.

\begin{tabular}{llll}
\hline E1 & & & 1,$40 ; 00,00$ \\
E2 & add & $4 ; 26,40$ & 1,$44 ; 26,40$ \\
E3 & add & $4 ; 26,40$ & 1,$48 ; 53,20$ \\
E4 & add & $4 ; 26,40$ & 1,$53 ; 20,00$ \\
E5 & add & $4 ; 26,40$ & 1,$57 ; 46,40$ \\
E6 & add & $4 ; 26,40$ & 2,$02 ; 13,20$ \\
E7 & add & $4 ; 26,40$ & 2,$06 ; 40,00$ \\
E8 & add & $4 ; 26,40$ & 2,$11 ; 06,40$ \\
E9 & add & $4 ; 26,40$ & 2,$15 ; 33,20$ \\
E10 & add & $4 ; 26,40$ & 2,$20 ; 00,00$ \\
E11 & subtract & $4 ; 26,40$ & 2,$15 ; 33,20$ \\
E12 & subtract & $4 ; 26,40$ & 2,$11 ; 06,40$ \\
E13 & subtract & $4 ; 26,40$ & 2,$06 ; 40,00$ \\
E14 & subtract & $4 ; 26,40$ & 2,$02 ; 13,20$ \\
E15 & subtract & $4 ; 26,40$ & 1,$57 ; 46,40$ \\
E16 & subtract & $4 ; 26,40$ & 1,$53 ; 20,00$ \\
E17 & subtract & $4 ; 26,40$ & 1,$48 ; 53,20$ \\
E18 & subtract & $4 ; 26,40$ & 1,$44 ; 26,40$ \\
E19 & subtract & $4 ; 26,40$ & 1,$40 ; 00,00$ \\
E20 & & & 1,$40 ; 00,00$ \\
\hline
\end{tabular}

and which can be used during several cycles of the SC scheme. The TU 11 method of predicting eclipse times can be seen as an early formation of theory. The method connects the timeshift between eclipses situated 1 Saros apart with the length of night and the actual month. This connection leads to another numerical function-the TU 11 zigzag function-which fits nature about as well as the 'simple 18-year function'. Thus, we have evidence for a theoretical treatment of the duration of the Saros that uses the month and length of night as the independent variable. In other words, it was the solar component of the variation in the Saros length which was modeled by the TU 11 function.

We now return to the simple 18-year function and the question of its date.

In Figure 5, the thin line shows the duration of the Saros tabulated for consecutive full moons - this thin line displays the typical structure of the superposition of two periodic functions with different amplitudes and periods. From such a curve, we can easily find the periods and amplitudes of the two components, ${ }^{21}$ though this was not the Babylonian method for separating out these factors. On the top of this curve, the thicker line shows the duration of the Saros, measured at consecutive EPs around the descending node, i.e. the Saros length measured at intervals of 12 and occasionally 11 months (marked by a circle). This curve is quite irregular and not easy to 'read'. The same is true for the sequence of numbers behind the curve. But as we have seen, this curve can be-and wasapproximated by a linear zigzag function with the period of a Saros. In other words, the sequence of numbers was modeled by numbers increasing (or decreasing) by $5 u \check{s}$ (and by $4 ; 26,40$ in the hypothetical TU 11 function). 


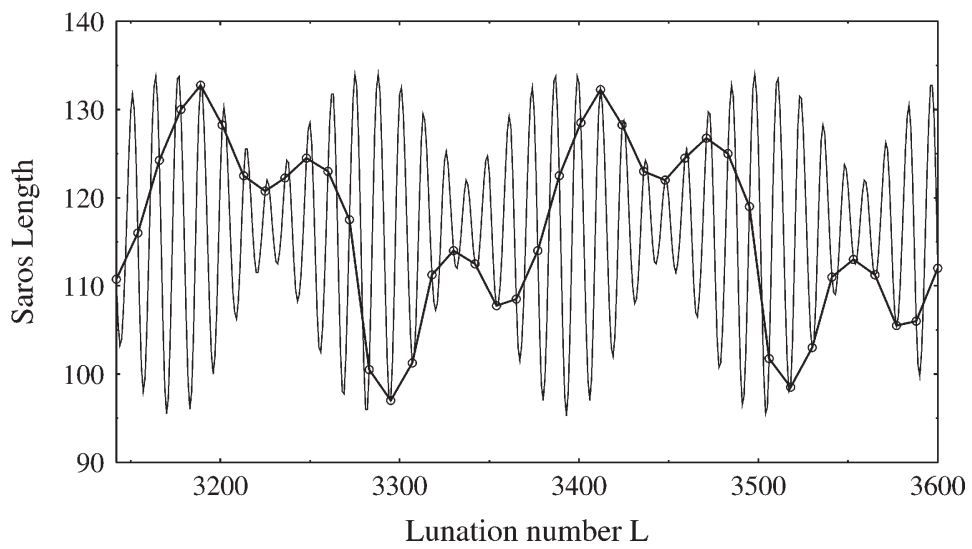

Fig. 5. Starting with the full moon 6 February 747 BC $(L=3,142)$, for 500 consecutive full moons, the duration of the Saros, given as function of the lunation number, is drawn with thin line. Those full moons which occur in the Saros cycle scheme as odd eclipse possibilities (EPs) are marked by circles and connected by a heavy line. The heavy curve is hence 'our Saros length curve', i.e. Saros length curve for odd EPs.

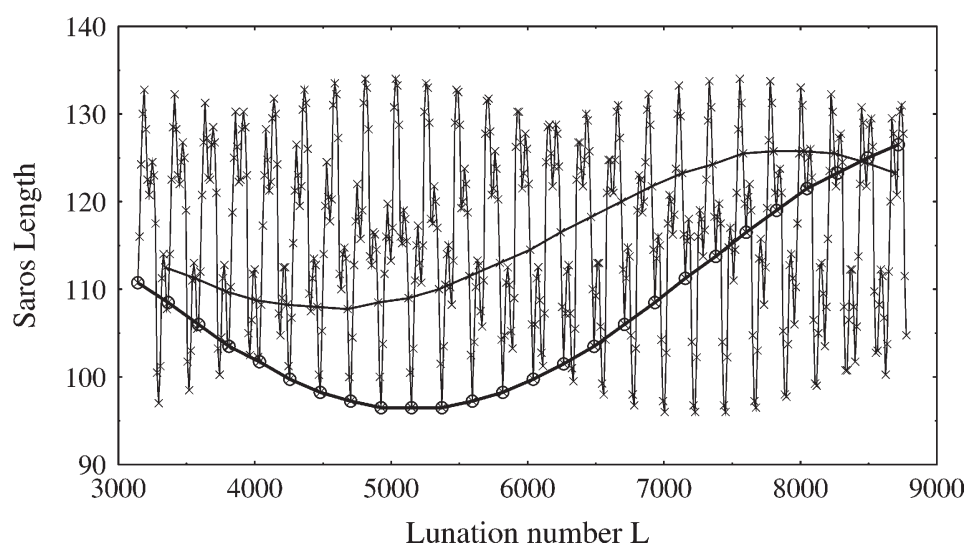

Fig. 6. The Saros length given for all 'odd' eclipse possibilities (EPs) during the first 25 cycles of the Saros cycle (SC) scheme, i.e. from 6 February 747 BC (= SC 1) until 8 June 297 BC (=SC 26), is drawn with a thin line. On the top of this curve, the first EPs for all 25 columns of the SC scheme are marked by a circle and connected by the thick line. The second thick line shows the Saros length from EP 35, i.e. the EPs at intervals of a Saros stemming from the EPs in line 35 of the scheme.

In Figure 6, for 25 Saroi we show our Saros length curve (i.e. the duration of the Saros at odd EPs) by the thin line. On the top of this curve, we have marked those EPs stemming from the first line of the SC scheme by circles connected by a thick line. Similarly, we have marked the EPs listed in line 35 just by a thick line. We concentrate on the curve through the first EP of each SC. Note that starting by SC 5 (at $L=4034$ ) and 


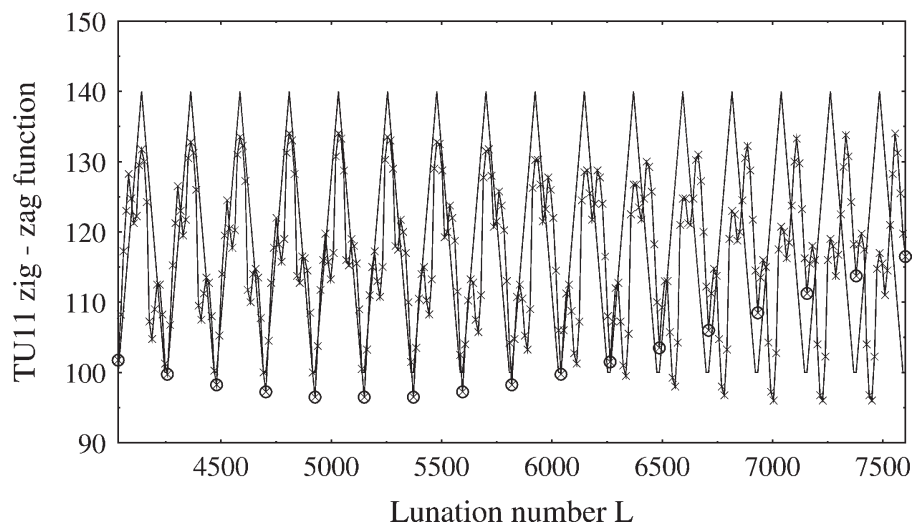

Fig. 7. Comparison between the Saros length and the TU 11 Saros length zigzag function (as in Figure 4). In this figure, for the 16 Saroi from 21 March 675 BC until 26 April 369 BC.

ending by SC 14 (at $L=6041$ ), the circle is situated at a local minimum of the curve. During these 9 Saroi, the Saros length curve can be nicely approximated by a linear zigzag function starting with minimum at EP 1. With time, the circles move on the curve- this is a consequence of the fact that the Saros is about 11 days longer than 18 years. In Saroi 1 and 2, the first EP is situated well after a minimum, and in Saros 18 (at $L=6,933$ ), it is situated before the local minimum. This explains why our zigzag function (which was constructed from the months of EPs in SC 8) only gives a good approximation to the Saros length curve during some 8 Saroi. After that period of time, one will have to construct a new zigzag function based on the months given, e.g. in column 15 of the SC scheme.

Figure 7 compares the Saros length curve with the TU 11 zigzag function again; here for a longer period of time than was the case in Figure 4-from SC 5 to SC 21. It illustrates the slow displacement of the first EPs (marked in the figure by circles) with respect to local minima of the Saros length curve. We see here that the TU 11 zigzag function only agrees well with the Saros length curve for 8 Saroi.

In the reconstruction of the TU 11 zigzag function for the Saros length, we had chosen SC 8 , and we got a zigzag curve starting at a minimum. This zigzag function approximates nature (locally) quite well during some 8 Saroi around SC 8. Had we chosen SC 20, we would have ended up with a zigzag function with the same extrema, but starting with EP 1 at the middle of the descending branch. The TU 11 method for predicting times of eclipses models nature well. Since the duration of the Saros is linked to the actual month, the slow shift of the Saros with respect to 18 years is implicitly incorporated in this method. If we are correct in our assumption that the TU 11 method for predicting eclipses gave rise to the 'TU 11 zigzag function', then we have here an example of a function for which the starting conditions would have to be determined (locally) for each given period of time. 
In this case, we even know how the new starting points of the proposed zigzag function were found (unlike the zigzag functions found in lunar System B).

Our insight into how the position of the (first) EP changes with time can be used for dating purposes. If we know the value of EP 1 for an approximating zigzag function and we also know if it is situated on the descending or ascending branch, then we can find the date at which this function gives an optimal fit to the Saros length curve.

In the beginning of our paper, we speculated that the 'strange numbers' $(1,40,2,10$, and 1,50 which could be attached to eclipses in lines 1, 2, and 35 of the SC scheme) possibly refer to the Saros length to be used for the respective lines of the SC scheme. We also pointed at their likely connection to the simple 18-year function. If these suppositions are correct, we have a means of dating them-just by figuring out where on the approximating zigzag function the number attached to EP 1 (or EP 2 or EP 35) is situated.

That the 'strange numbers' have their origin in the simple 18-year function is supported by the fact that it is possible to continue and connect them by means of the numbers of the simple 18-year function. For the 'odd' EPs starting with 1,40 in line 1, we propose the following series of 19 Saros length numbers: $\mathbf{1 , 4 0} \quad 1,35 \quad 1,35 \quad 1,40 \quad 1,45 \quad 1,50 \quad 1,55 \quad 2,00$ $2,052,102,152,20 \quad 2,15 \quad 2,10 \quad 2,05 \quad 2,00 \quad 1,55 \quad \mathbf{1 , 5 0} \quad 1,45$, and we are back at 1,40 for the first line of SC 2. The only other possibility of connecting the 'strange numbers' would be to repeat the maximum value of 2,20 instead of taking the minimum 1,35 twice:

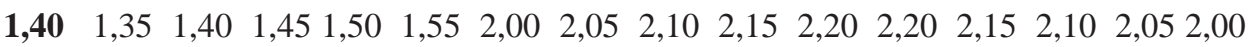
$1,551,501,45$, and we are again back at 1,40 for the first line of SC 2 . It is evident, however, that only the first series can be used. We know that EP 2 has the number 2,10 which is situated two EPs before a maximum - only by repeating the minimum will the two Saros curves be out of phase by nine lines, as they should be.

Assuming that this reconstruction is correct (and we can see no other plausible possibilities), we can now date both the 'strange numbers' and the simple 18-year function. Figure 8 tells us that the Saros length for line 1 will be exactly 1,40 (=100) at the beginning of SC 6 and SC 14. The best fit for 1,50 (=110) for line 35 is at the end of SC 3 and SC 10. For the 'even' EPs in line 2, we also have two optimal fits for the number 2,10 (=130): at the beginning of SC 8 and SC 14. If we combine these possible fits, we get somewhere between SC 4 and SC 8 or somewhere between SC 11 and SC 14.

Only the last possibility agree to the fact that the first EP in the scheme shall be situated on the descending branch (i.e. before the minimum) of the Saros length zigzag function. This is the case for the first EP in SC 13 or SC 14 but not in SC 6. In SC 6, the number 1,40 of the first EP must be situated on the ascending branch, with the consequence that the number attached to EP 37 would have to be 1,35. This is, however, impossible, since we know the number 1,50 of EP 35.

We sum up: the 'strange numbers' and the simple 18-year function fit optimally to the time around $531 \mathrm{BC}$, and therefore we conclude that this might be the approximate period when they were constructed and used. However, this dating and any conclusions drawn 


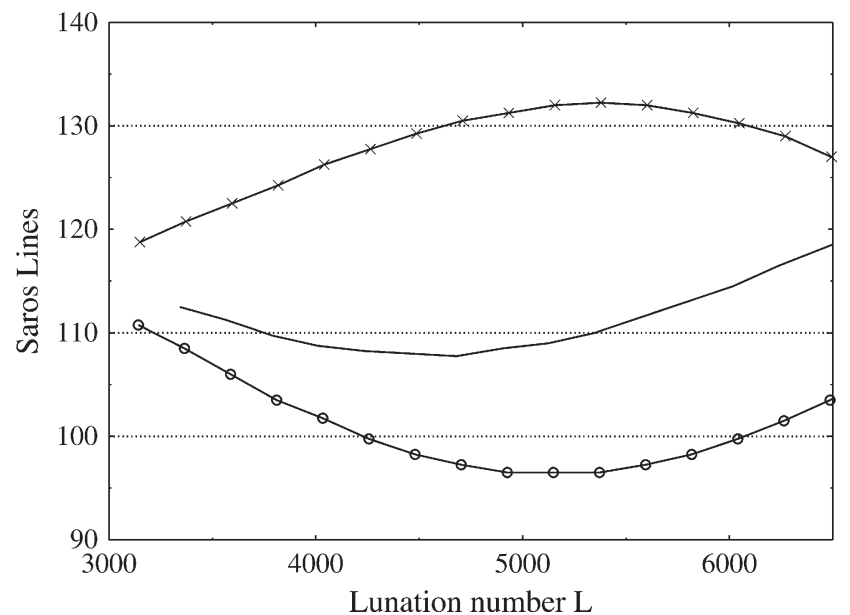

Fig. 8. The Saros length tabulated over 16 Saroi at intervals of 1 Saros. The Saros lengths at eclipse possibilities from line 1 of the Saros cycle scheme are marked by circles and connected by a thick line. Those stemming from line 2 are marked by crosses and connected by a line, and finally those from line 35 are just connected by a line. The circles mark the beginning of SC $1, \mathrm{SC} 2, \ldots, \mathrm{SC} 16$.

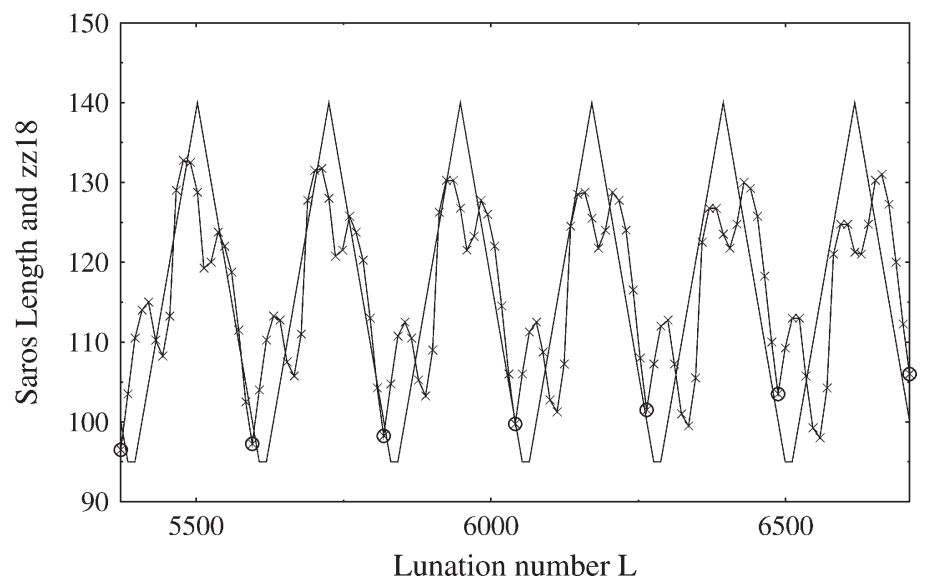

Fig. 9. The Saros length, tabulated over 6 Saroi at consecutive 'odd' eclipse possibilities (EPs), is shown as crosses connected by a thin line. We start at $L=5,149$ which is the first EP of Saros cycle (SC) 11 and end with $L=6,710$ which is the the first EP of SC 17. This curve is well approximated by the simple 18-year zigzag function starting with 1,40 $1,35 \quad 1,35 \quad 1,40$ etc. which is the version correct for that time.

upon it must be regarded as tentative. It is not possible to date the TU 11 method, since it is not 'frozen' but instead correctly pays regard to the slow change of solar anomaly over time.

In Figure 1, we had compared the Saros length with an arbitrary version of the simple 18-year zigzag function (called zz18). We had let it start at a minimum and got a 
Table 7. The duration of the Saros according to different models.

\begin{tabular}{llccc}
\hline SC 14 & Month & $\begin{array}{c}\text { TU11 } \\
\text { duration }\end{array}$ & $\begin{array}{c}\text { TU11 zigzag } \\
\text { function }\end{array}$ & $\begin{array}{c}\text { Simple } \\
18 \text {-year } \\
\text { function }\end{array}$ \\
\hline EP 1 & III & 1,$40 ; 00$ & 1,$44 ; 26,40$ & 1,40 \\
EP 3 & III & 1,$40 ; 00$ & 1,$40 ; 00,00$ & 1,35 \\
EP 5 & III & 1,$40 ; 00$ & 1,$40 ; 00,00$ & 1,35 \\
EP 7 & II & 1,$46 ; 40$ & 1,$44 ; 26,40$ & 1,40 \\
EP 9 & I & 1,$53 ; 20$ & 1,$48 ; 53,20$ & 1,45 \\
EP 11 & XII & 2,$00 ; 00$ & 1,$53 ; 20,00$ & 1,50 \\
EP 13 & XII & 2,$00 ; 00$ & 1,$57 ; 46,40$ & 1,55 \\
EP 15 & XII & 2,$00 ; 00$ & 2,$02 ; 13,20$ & 2,00 \\
EP 17 & XI & 2,$06 ; 40$ & 2,$06 ; 40,00$ & 2,05 \\
EP 19 & X & 2,$13 ; 20$ & 2,$11 ; 06,40$ & 2,10 \\
EP 21 & X & 2,$13 ; 20$ & 2,$15 ; 33,20$ & 2,15 \\
EP 23 & IX & 2,$20 ; 00$ & 2,$20 ; 00,00$ & 2,20 \\
EP 25 & VIII & 2,$13 ; 20$ & 2,$15 ; 33,20$ & 2,15 \\
EP 27 & VIII & 2,$13 ; 20$ & 2,$11 ; 06,40$ & 2,10 \\
EP 29 & VIII & 2,$13 ; 20$ & 2,$06 ; 40,00$ & 2,05 \\
EP 31 & VI & 2,$00 ; 00$ & 2,$02 ; 13,20$ & 2,00 \\
EP 33 & VI & 2,$00 ; 00$ & 1,$57 ; 46,40$ & 1,55 \\
EP 35 & V & 1,$53 ; 20$ & 1,$53 ; 20,00$ & 1,50 \\
EP 37 & V & 1,$53 ; 20$ & 1,$48 ; 53,20$ & 1,45 \\
EP 1 & & & 1,$44 ; 26,40$ & 1,40 \\
\hline
\end{tabular}

nice fit around SC 8. This only indicated that, in principle, the function zz18 could be a useful-i.e. rather precise - approximation to the Saros length, but we could not determine a date at which it was used. Now we know the time (SC 13 or SC 14) at which the 'strange numbers' must have been determined, and we know which EP correspond to the maximum and minimum of the zigzag function. So now-for the time around SC 13-we can compare the Saros length calculated for 'odd EPs', with the simple 18-year zigzag function starting with 1,40 $1,35 \quad 1,35 \quad 1,40$ ( . . and ending with $\mathbf{1 , 5 0} 1,45$ ) which is correct for that time. This is done in Figure 9.

Finally, we will compare the TU 11 zigzag function for the time around $530 \mathrm{BC}$ with the simple 18-year zigzag function. We get the TU 11 zigzag function to be used for that time by smoothing out the number sequence given in column 3 of Table 7. Columns 1 and 2 give the odd EP numbers together with the actual month for SC 14. Column 3 lists the Saros length of the respective months found according to the TU 11 method. Column 4 gives the TU 11 zigzag function, and column 5 lists the values of the simple 18-year function. The Babylonian months given in SC 13 would deliver exactly the same zigzag function as the one from SC 14. We have here chosen to use SC 14 since it has month IX giving the maximal Saros length of 2,20 uš, while in SC 13, month IX does not occur.

We conclude that the TU 11 method approximates the duration of the Saros surprisingly well and that the TU 11 zigzag function determined for the time around $530 \mathrm{BC}$ is almost 
identical with that simple 18-year function which connects the 'strange numbers'. The question which of the two function was the primary is still unresolved.

\section{Acknowledgements}

We both would like to thank the British Academy for the generous provision of a British Academy Visiting Fellowship which enabled this collaboration during Lis Brack-Bernsen's 3-week visit to England. We thank M. Brack for providing the computations behind the figures, and John Britton for useful comments and suggestions to this paper. Lis BrackBernsen further thanks the Deutsche Forschungsgemeinschaft for supporting her research, while John Steele thanks the Leverhulme Trust and the Royal Society for their generous provisions of research fellowships.

\section{BIBLIOGRAPHY}

Al-Rawi, F. N. H., and George, A. R.

1991: "Enuma Anu Enlil XIV and other early astronomical tables", Archiv für Orientforschung 37, 52-69.

Brack-Bernsen, L.

1980: "Some Investigations on the Ephemerides of the Babylonian Moon Texts, System A", Centaurus 24, 36-50.

2002: "Predictions of Lunar Phenomena in Babylonian Astronomy" in J. M. Steele and A. Imhausen (eds.), Under One Sky: Astronomy and Mathematics in the Ancient Near East, Ugarit-Verlag, Münster, pp. 5-19.

2005: “The 'Days in Excess' from MUL.APIN: On the 'First Intercalation' and 'Waterclock' Schemes from MUL.APIN", Centaurus 47, 1-29.

Brack-Bernsen, L., and Hunger, $\mathrm{H}$.

2002: "TU 11, A Collection of Rules for the Prediction of Lunar Phases and of Month Length", SCIAMVS 3, 3-90.

Britton, J. P.

1989: “An Early Function for Eclipse Magnitudes in Babylonian Astronomy," Centaurus 32, 1-52.

Brown, D

2000: Mesopotamian Planetary Astronomy-Astrology, Styx, Gronignen.

Goldstine, H. H.

1973: New and Full Moons 1001 BC to AD 1651, American Philosophical Society, Philadelphia, PA.

Huber, P. J., and S. de Meis

2004: Babylonian Eclipse Observations from 750 BC to 1 BC, IsIAO-Mimesis, Milano.

Hunger, $\mathrm{H}$.

2001: Astronomical Diaries and Related Texts from Babylonia. Volume 5: Lunar and Planetary Texts, Österreichische Akademie der Wissenschaften, Wien.

Hunger, H., and Pingree, D.

1989: "MUL.APIN: An Astronomical Compendium in Cuneiform", Archiv für Orientforschung 24, Verlag Ferdinand Berger, Horn, Austria.

Moesgaard, K. P.

1980: “The Full Moon Serpent: A Foundation Stone of Ancient Astronomy?”, Centaurus 24, 51-96.

Neugebauer, O.

1955: Astronomical Cuneiform Texts, Lund Humphries, London. 
Steele, J. M.

2000a: "Eclipse Prediction in Mesopotamia", Archive for History of Exact Science 54, 421-454.

2000b: Observations and Predictions of Eclipse Times by Early Astronomers, Kluwer Academic Publishers, London.

2002: "A Simple Function for the Length of the Saros in Babylonian Astronomy" in J. M. Steele and A. Imhausen (eds.), Under One Sky: Astronomy and Mathematics in the Ancient Near East, Ugarit-Verlag, Münster, pp. 405-420.

Steele, J. M., and F. R. Stephenson

1997: "Lunar Eclipse Times Predicted by the Babylonians", Journal for the History of Astronomy 28, 119-131.

Walker, C. B. F.

1997: "Achaemenid Chronology and the Babylonian Sources", in J. Curtis (ed.). Mesopotamia and Iran in the Persian Period: Conquest and Imperialism 539-331 BC, British Museum Press, London, pp. 17-25.

\section{NOTES}

1. See Steele (2000a) for further details.

2. LBAT 1413, published as Text No. 1 in Hunger (2001).

3. LBAT $^{*} 1414,1415+1416+1417$, and * 1419, published as Texts Nos. $2-4$ in Hunger (2001). See the discussion by Steele in the Appendix to that volume, Walker (1997), and Huber and de Meis (2004, pp. 1-6).

4. Moesgaard (1980), Britton (1989).

5. The situation is slightly more complicated since times were measured relative to sunrise or sunset, and so corrections would have to be applied to take into account the change in daylength.

6. Somewhat surprisingly, the variation in the length of the Saros is largely a result of the variation in solar, rather than lunar, anomaly. See Brack-Bernsen (1980).

7. Reported in the appendix by Steele to Hunger (2001), to which a reference to Brown's publication should therefore be added to footnote 4.

8. For example, the Saros scheme as known from the large compilation breaks down two cycles before $747 \mathrm{BC}$ with the eclipse of 30 June $773 \mathrm{BC}$; had there been systematic eclipse records from this period then the Saros scheme would very likely been aligned to accommodate these. We thank John Britton for this insight.

9. See Steele (2002).

10. For a transcription of BM 45861 which preserves the layout of the tablet, see Steele (2002). The text begins with 2,5 increasing and continues to 2,10 decreasing with a single minimum.

11. See figure 3 of Steele (2002).

12. See figure 4 of Steele (2002).

13. A similar plot for the even-numbered eclipses would simply be a mirror image of this plot.

14. Steele (2000b, pp. 57-68).

15. Hunger and Pingree (1989, pp. 75-76 or pp. 101-107).

16. Al-Rawi and George (1991).

17. See Britton (1989, pp. 29-35).

18. This lunar scheme (usually called the 'waterclock' scheme) gives the daily retardation of the moon for the 1 st and 15 th day of each month in the ideal year; i.e. it gives the daily retardation of the fulland new moon during the year. See Brack-Bernsen (2005).

19. See Brack-Bernsen (2005).

20. See Brack-Bernsen (2002).

21. See Brack-Bernsen (1980). 
Copyright of Centaurus is the property of Blackwell Publishing Limited. The copyright in an individual article may be maintained by the author in certain cases. Content may not be copied or emailed to multiple sites or posted to a listserv without the copyright holder's express written permission. However, users may print, download, or email articles for individual use. 